\title{
FIRST TESTS OF THE TRAPPED ION SOURCE
}

\author{
V. Variale, V. Valentino, INFN sez. di Bari \\ G.Brautti, A. Boggia, A. Raino', Dipartimento di Fisica e INFN sez. di Bari
}

\begin{abstract}
Recently the detailed design and the construction problems of a Trapped Ion Source (TIS) have been presented in ref.[1,2]. In practice, TIS can be seen as a modified version of an Electron Beam Ion Trap (EBIT) or of an Electron Beam Ion Source (EBIS). The main new feature of TIS, with respect to an EBIS (or EBIT), is the transverse ion confinement given by a quadrupole field instead of the electron beam space charge. One can foresee that TIS could overcome some drawbacks of the EBIT and EBIS making it a more flexible device. In this paper a detailed discussion on the radio frequency containment of high charge state ions with the first rf containment test will be presented.
\end{abstract}

\section{INTRODUCTION}

TIS is a new type of source capable, in principle, of producing very highly charged ions and, at the same time, it is a radio frequency (rf) quadrupole linear trap suitable to study the interaction of the trapped ions (or charged microparticles) with electrons, high energy particles or laser beams. In practice, it is a modified version of an Electron Beam Ion Source (EBIS). TIS, in fact, is an EBIS where an rf quadrupole field has been added to contain transversally the ion produced by the electron beam.

Recently some proposals to use EBIS type sources as charge state breeder for radioactive ion beams extracted as singly charged ions with the isotope separator on-line (ISOL) technique have been suggested [3]. In fact, for efficient acceleration by compact linear accelerators, ions with typical mass ratios of $1 / 9$ are required. However, after the high charge state ions have been produced, a mass separator is required before the ion beam acceleration. In this case, the use of TIS instead of an EBIS, could allow to accelerate radioactive ion beams directly at the exit of the TIS source. In fact, having TIS a selective ion containment given by the rf quadrupoles, it is, at the same time, an ion source and an ion mass separator.

Among the goal of TIS, other than the production of highly charged particles, it can be foreseen ion cooling studies, analysis of macromolecules and, also, "dust targets" for high energy accelerators. The status report of the project with a detailed discussion on the rf ion containment system will mainly be presented.

\section{TIS DESIGN}

TIS design has already presented in ref. [1,2], but here, for clarity sake, its main features will be recalled. In fig.1 a cutaway drawing of the ion source mechanical design and its operation scheme is shown. From the fig.1b) it can be seen the electron gun that generates the electron beam needed to ionize the atoms. Since an electron gun designed and built for another experiment is intended to use for TIS experiment a couple of iris are been used to match the electron beam emittance to the acceptance of the TIS transport channel.

The transport channel that drives the electron beam until to the collector is made of two 90 degree bending magnets (BM) and several quadrupole doublets of new design as shown in more details in ref. [2]. The main new feature of TIS, with respect to an EBIT (or EBIS), is the adding of radial ion confinement of the rf quadrupoles to the potential well of the eb space charge when it is on. When the eb is off (the eb will be pulsed) only the desired ions will remain trapped.

In the longitudinal direction the containment is obtained by two repelling electrodes placed at the edges of the quadrupole electrodes. These electrodes can be pulsed to pull-out the trapped ions for external use (e.g. acceleration).

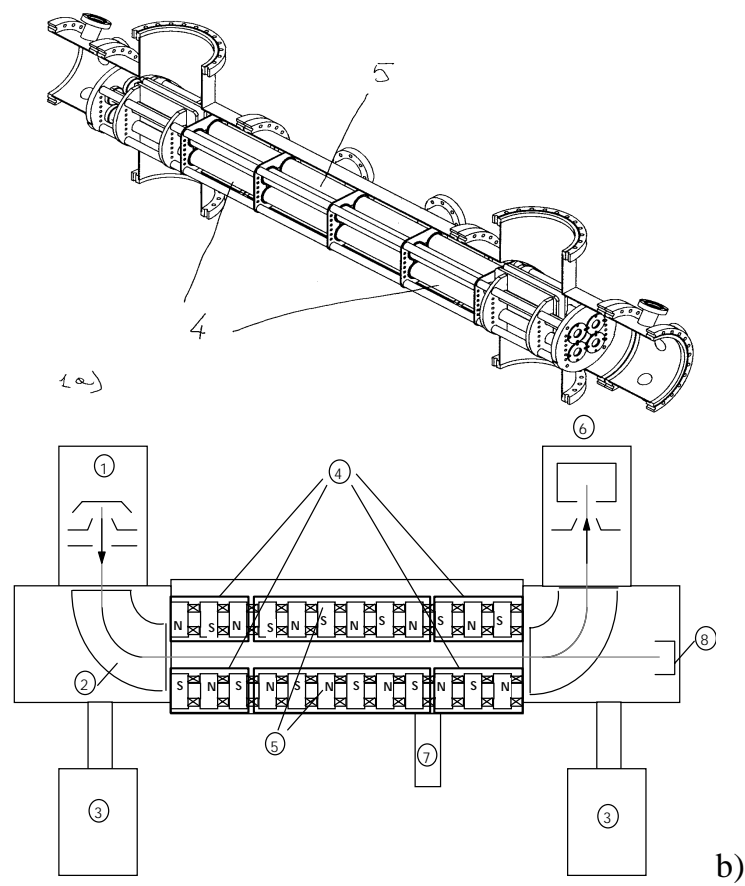

Fig.1a) cutaway view of the TIS mechanical design; 1b) Operation scheme of TIS: 1) electron gun, 2) bending 
magnet, 3) vacuum pump, 4) static potential electrodes $\mathrm{U}_{0}$ for longitudinal ion trapping, 5) rf quadrupole electrodes with inside the focusing magnetic quadrupoles, 6) electron collector, 7) gas-inlet, 8) ion collector.

In its normal operation, the electron beam pulse is injected transversally (see fig 1b) in the trap and then bent in the axial direction. Transversally too, in the center of the trap, vapor or powder can be injected, by a valve gas jet, that it is ionized and confined by the rf quadrupole field.

\section{RADIO FREQUENCY CONTAINEMENT}

The TIS eb transport line has been described in detail in ref [2]. In this paper we will present the rf system utilized to contain, transversally, the wanted ions with different charge states produced by the electron beam.

In a EBIS, the ion containment in the transverse planes is obtained by the potential well due to the space charge of the ionizing electron beam and longitudinally from the potential set by the electrodes placed on the edges of the ionization region. In these conditions the residual gas ions being in the vacuum chamber can be trapped together the wanted ions. For this reason EBIS sources require very high vacuum (about $10^{-11}$ Torr) and at the same time very dense energetic electron beams [3]. Furthermore the cold electrons coming from the ionization can be trapped by the ion space charge increasing, in this way, the probability of the recombination process. In TIS, to avoid those drawbacks, the adding of the rf quadrupole field is provided by 4 cylindrical shaped electrodes, see fig.1). Although, infinite hyperbolically shaped electrodes are needed for a pure quadrupole field, an appropriate chose of the ratio between the electrode cylinder radius and their distance from the symmetry axis can be chosen for a very good optimization of the quadrupole field. In ref. [1] we have found a very good optimization for a cylinder radius of $2.2 \mathrm{~cm}$ and a distance from the symmetry axis of $2 \mathrm{~cm}$. In fact these parameters give a quadrupole Fourier coefficient, normalized to the sextupole coefficient which is the higher among the other multipole coefficients of $2.7 \times 10^{4}$.

The equations of motion, at the presence of the potential given by these electrodes, in the transverse planes, can be written in Mathieu's equation form:

$$
\begin{aligned}
& \frac{d^{2} X}{d \theta^{2}}+(a-2 q \cos 2 \theta) X=0 \\
a= & \pm \frac{8 e U}{m r_{0}^{2} \omega^{2}} \\
q= & \mu \frac{4 e V}{m r_{0}^{2} \omega^{2}}
\end{aligned}
$$

and where: $\mathrm{X} \rightarrow \mathrm{x}, \mathrm{y} ; \theta \rightarrow \omega \mathrm{t} / 2$, and the upper and lower signs correspond to differential equations in $\mathrm{x}$ and $\mathrm{y}$, respectively. The applied voltage has a dc component $U$ plus an rf voltage $V$ with the driving frequency $\omega$.

The Mathieu equation solution can be stable or unstable. Whether stability exists depends only on the parameters $a$ and $q$ and not on the initial parameters of

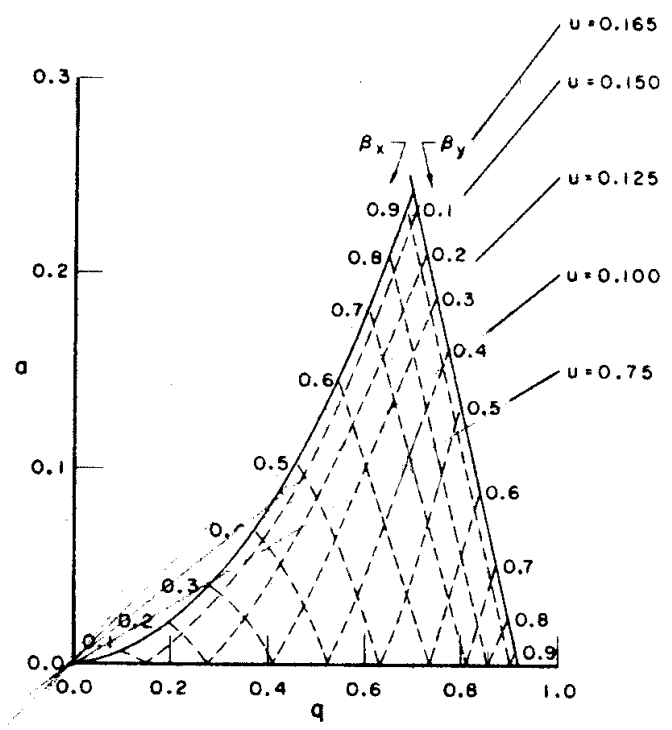

Fig.2 The $a-q$ stability diagram. The positions of various operating lines $U / V=$ Const. are also indicated.

the ion motion. In fig. 2 is shown the region of simultaneus stability in both the transverse planes $\mathrm{x}$ and y. It can be noticed that a given set of applied voltages $U$ and $V$ uniquely determines the relative values of $a$ and $q$ in the stability diagram. If we Define $u=|U| /|V|=a / 2 q$ a typical straight line $a=2 q u$ can be drawn as shown in fig.2. The portion of this line passing through the stable region of the diagram determines the range of ionic $\mathrm{e} / \mathrm{m}$ values which will have stable trajectories through the quadrupole field. As the $U / V$ ratio is increased, the intersected stability region becomes narrower and narrower, so that the device becomes more highly mass selective, but at the same time the $\mathrm{x}$ and $\mathrm{y}$ acceptances of the trap become smaller. Furthermore, it must be noticed that, for a chosen $U / V$ ratio, the ions with stable $\mathrm{e} / \mathrm{m}$ values can be scanned by varying the magnitude of both $\mathrm{U}$ and $\mathrm{V}$ simultaneously or by varying the applied frequency $\omega$.

The operating point in the stability diagram $a-q$ can be chosen in such a way to satisfy the required charge state on the output ion. In some cases it could not be necessary the $e / m$ scanning as mentioned before. As an example we consider the case of using TIS to produce $\mathrm{Ar}^{+4}$, which is the element we are using in our first tests. If we choose a line (see fig.2)with a low $U / V$ ratio, renouncing to a large $\mathrm{e} / \mathrm{m}$ resolving power, will have a large range of stability . Let us choose as lower $q$ (the $q$ value given by the interception of the line $a=2 q u$ with the left part of 
stability triangle) the value 0.2 , from the definition of $q$ and using $\mathrm{V}=1 \mathrm{kV}$ we obtain the $\mathrm{rf}$ frequency $\mathrm{v}=1.7$ MHz. The corresponding $a$ value is (see fig.2) 0.02 then $u=a / 2 q=0.05$. Following the operation line $a=2 q u$, the higher $q$ value 0.82 is intercepted on the stability diagram and at this point an $A / Z=10\left(\mathrm{Ar}^{+4}\right)$ will also have a stable trajectory in TIS. In conclusion, with the choice of the above parameters we have stable trajectories from $\mathrm{Ar}^{+1}$ to $\mathrm{Ar}^{+4}$. In these conditions of low resolving power, we expect that other elements with $e / m$ inside the stability diagram could be trapped with wanted ions. The other elements present with $\mathrm{Ar}$ in the vacuum tank are, in general, the component of the residual gas. The tank residual gas has been analyzed and was mainly composed by $\mathrm{N}_{2}(\mathrm{~A}=28)$, water $(\mathrm{A}=18), \mathrm{O}(\mathrm{A}=16), \mathrm{N}(\mathrm{A}=14), \mathrm{H}_{2}$ $(A=2)$. By using the above parameters $(\mathrm{V}=1.7 \mathrm{MHz}, \mathrm{V}=1$ $\mathrm{kV}, \mathrm{U}=50 \mathrm{~V}$ ) we have found that also the $e / m$ of $\mathrm{N}_{2}^{+1}$, $\mathrm{N}_{2}^{+2}, \mathrm{H}_{2} \mathrm{O}^{+1}$ and $\mathrm{N}^{+1}$ are in the stable region while the other $\mathrm{e} / \mathrm{m}$ values lead to unstable trajectories.

The preliminary rf test done on TIS have had the aim to check the ion containment capability and the quadrupole symmetry of the potential well. In fig. 3 is shown the residual gas plasma containment of the rf cylindrical shaped electrodes. The parameters used has been: $\mathrm{v}=2.5 \mathrm{MHz}, \mathrm{V}=0.6 \mathrm{kV}$ and $\mathrm{U}=50 \mathrm{~V}$ giving stability for ions with $\mathrm{A}>2$.

From fig.3, it can be seen a slight deformation of the quadrupole symmetry caused by a defect in feeding the rf electrodes. In fact, we did not have the exact same voltage, in opposition of phase, on the adjacent electrodes. We are correcting this defect with a better matching circuit having a more balanced transformer.

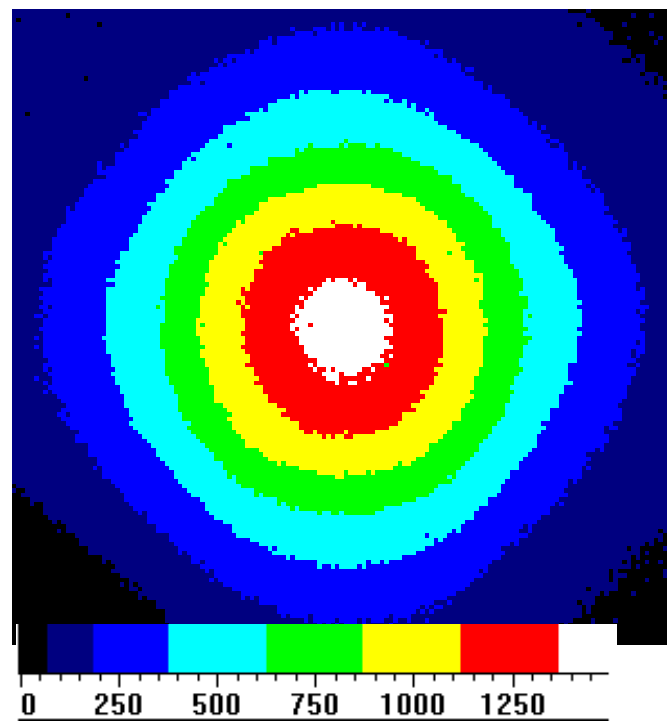

Fig.3 Plasma containment of the rf quadrupole electrodes as seen by a CCD camera placed at the end of the quadrupole axis. The intensity (ion density) levels are shown in the lower left corner.

\section{STATUS OF THE PROJECT AND CONCLUSION}

The mechanical design and the computer simulations of the device has been concluded. The electron gun test has been carried out at low voltage and, by measuring the electron current with a Faraday cup, a perveance of about $0.2 \mu \mathrm{P}$ has been found.

The construction and the test of all the quadrupole doublets needed for the eb focusing have been done. The $90^{\circ}$ bending magnets are also been constructed and tested.

Steering coils with Beam Position Monitor (BPM) has been placed behind the first and at the entrance of the second bending magnet. The system to excite the quadrupole doublet coils inside the rf cylindrical electrodes (as shown in ref.2) are also been tested. The vacuum chamber has also been tested and the cylinder shaped electrodes for the rf quadrupole field are also been tested.

In conclusion, we need only of the voltage pulser to drive the electron gun that will be available in the next month. After that we will be ready for the test of the electron beam transport from the gun to the collector and then for the ion productions and their trapping.

\section{REFERENCES}

[1] G. Brautti, A. Boggia, A. Rainò, V. Valentino, V. Variale "Trapped Ion Source", Proc. of PAC'97 Vancouver (1997).

[2] G. Brautti A. Boggia, A. Rainò, V. Valentino, V. Variale "Study of a Trapped Ion Source" proc. of EPAC98 Stockholm (1998)

[3] U: Köster, O. Kester and D. Habs, Rev. of Sci. Inst., Vol. 69, N.3 (1998), 1317.

[4] P. H. Dawson, Applied Charged Particle Optics, 13B, ed. A. Septier, pag. 173, Academic Press (1980) 